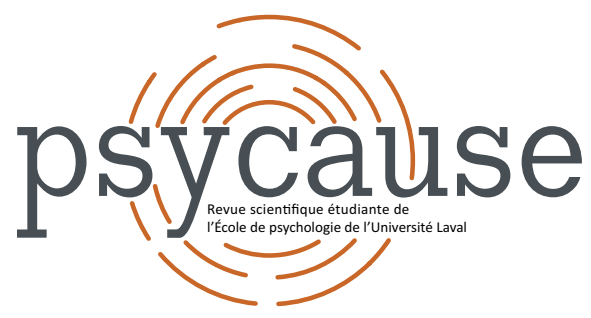

\title{
PSYCAUSE
}

Revue scientifique étudiante de l'École de psychologie de l'Université Laval

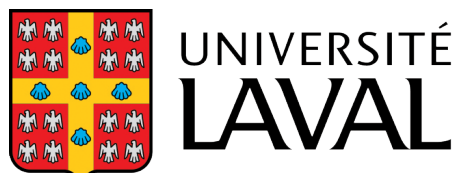

Faculté des sciences sociales École de psychologie

NOVEMBRE 2020 - VOL. $10 \mathrm{~N}^{\circ} 2$

\section{RELATION ENTRE LE FIGEMENT ET L'INHIBITION COMPORTEMENTALE CHEZ LE JEUNE ENFANT}

Jérôme GRAVEL ${ }^{1 *}$, Agnès ÉTHIER ${ }^{1}$, Lysandre PROVOST ${ }^{1}$ \& Michel BOIVIN ${ }^{1}$

${ }^{1}$ École de psychologie, Université Laval

*jerome.gravel.1@ulaval.ca

\section{Pour citer l'article}

Gravel, J., Éthier, A., Provost, L., \& Boivin, M. (2020). Relation entre le figement et l'inhibition comportementale chez le jeune enfant. Psycause: Revue scientifique étudiante de l'École de psychologie de l'Université Laval, 10(2), 23-24. 


\title{
RELATION ENTRE LE FIGEMENT ET L'INHIBITION COMPORTEMENTALE CHEZ LE JEUNE ENFANT
}

\author{
Jérôme GRAVEL ${ }^{1 *}$, Agnès ÉTHIER ${ }^{1}$, Lysandre PROVOST ${ }^{1}$ \& Michel BOIVIN¹ \\ 1 École de psychologie, Université Laval \\ *jerome.gravel.1@ulaval.ca
}

\section{Mots-clés : Figement, inhibition comportementale, adaptabilité, contribution génétique}

En réaction à des stimuli menaçants ou nouveaux, un éventail de réponses peut être observé dont le figement. Cette réponse est notamment caractérisée par une réduction de mobilité et une augmentation du tonus musculaire (Roelofs, 2017). L'inhibition motrice caractérisant le figement permet à l'organisme d'évaluer attentivement la menace et de sélectionner la réponse la plus adaptée (Roelofs, 2017). Cependant, l'aspect adaptatif de cette réponse dépend grandement de son contexte. En effet, un prolongement de cet état ou sa manifestation en l'absence d'une réelle menace peut signaler une faible adaptabilité (Buss, 2011). L'inhibition comportementale, un trait de tempérament proposé par Kagan, Reznick, Clarke, Snidman et Garcia-Coll (1984), s'apparente au figement puisqu'elle se manifeste également par un comportement passif et une inhibition motrice. Selon Buss (2011), il s'agit d'un type de tempérament très général référant à plusieurs dimensions, dont des aspects communicatifs (p. ex., pleurs) et comportementaux (p. ex., fuite). Ainsi, le figement et l'inhibition comportementale peuvent être perçus comme des stratégies d'adaptation passives caractérisées par une inhibition motrice et un déficit dans l'adaptation à des situations nouvelles ou menaçantes. Malgré ces éléments communs, la relation entre le figement et l'inhibition comportementale demeure méconnue (Hagenaars, Oitzl, \& Roelofs, 2014). D'ailleurs, la composante génétique de l'inhibition comportementale est établie dans la littérature (Garcia Coll, Kagan, \& Reznick, 1984), mais aucune étude de jumeaux n'a examiné celle du figement. Cette étude a ainsi trois objectifs, soit à documenter l'association entre le figement et l'inhibition comportementale chez le jeune enfant, à évaluer dans quelle mesure l'inhibition comportementale et le figement prédisent le trait d'adaptabilité et finalement à examiner les contributions génétiques au figement par la méthode des jumeaux.

\section{Méthode}

Les participants font partie de l'Étude des Jumeaux Nouveau-nés du Québec (ÉJNQ), une étude longitudinale prospective d'une cohorte de jumeaux nés dans la région de Montréal entre avril 1995 et décembre 1998. Pour la présente étude, I'adaptabilité, l'inhibition comportementale et le figement ont été mesurés respectivement auprès de 1130, 489 et 506 enfants âgés en moyenne de 19,6 mois.
Le tempérament de l'enfant a été évalué par le Infant Characteristics Questionnaire (ICQ; Bates, Freeland, \& Lounsbury, 1979) rempli par la personne qui connaît le mieux l'enfant, soit la mère dans $99 \%$ des cas. Dans l'ÉJNQ, trois items ont été retenus parmi les cinq du questionnaire pour mesurer l'adaptabilité de l'enfant: "Comment votre enfant réagit-il typiquement devant une nouvelle personne?», "Comment votre enfant réagit-il typiquement dans un nouvel environnement?» et "Comment votre enfant s'adapte-t-il éventuellement à de nouvelles expériences?». L'inhibition comportementale et le figement ont été évalués dans une situation de nouveauté qui consiste en une exposition de 140 secondes à un robot mécanique. La mère est présente lors de la confrontation, mais a pour consigne de ne pas interagir avec son enfant. À l'aide d'enregistrements des confrontations, l'inhibition comportementale a été codifiée préalablement à la présente étude par deux étudiantes de deuxième cycle alors que le figement a été codifié par l'équipe de recherche dirigée et une étudiante de deuxième cycle. Pour identifier un épisode de figement, les trois critères mandataires sont: une durée de figement minimale de trois secondes, une absence de vocalisations ainsi qu'une réduction de mobilité.

\section{Résultats et discussion}

Tout d'abord, une analyse factorielle a été effectuée afin de déterminer les différentes dimensions de l'inhibition comportementale. Elle révèle deux dimensions distinctes, soit les comportements d'évitement et les affects. Les résultats montrent que la corrélation entre le figement et les comportements d'évitement est significative et modérée, mais nulle et non significative entre le figement et les affects. Le figement étant corrélé avec la dimension des comportements d'évitement et non celle des affects, celui-ci semble lié à une dimension centrale du construit de l'inhibition comportementale plutôt qu'au construit global. Le figement serait davantage un indicateur de l'inhibition comportementale par sa relation avec les comportements d'évitement plutôt qu'un concept totalement distinct de l'inhibition comportementale.

Ensuite, les résultats d'une régression multiple montrent que les deux composantes de l'inhibition comportementale 


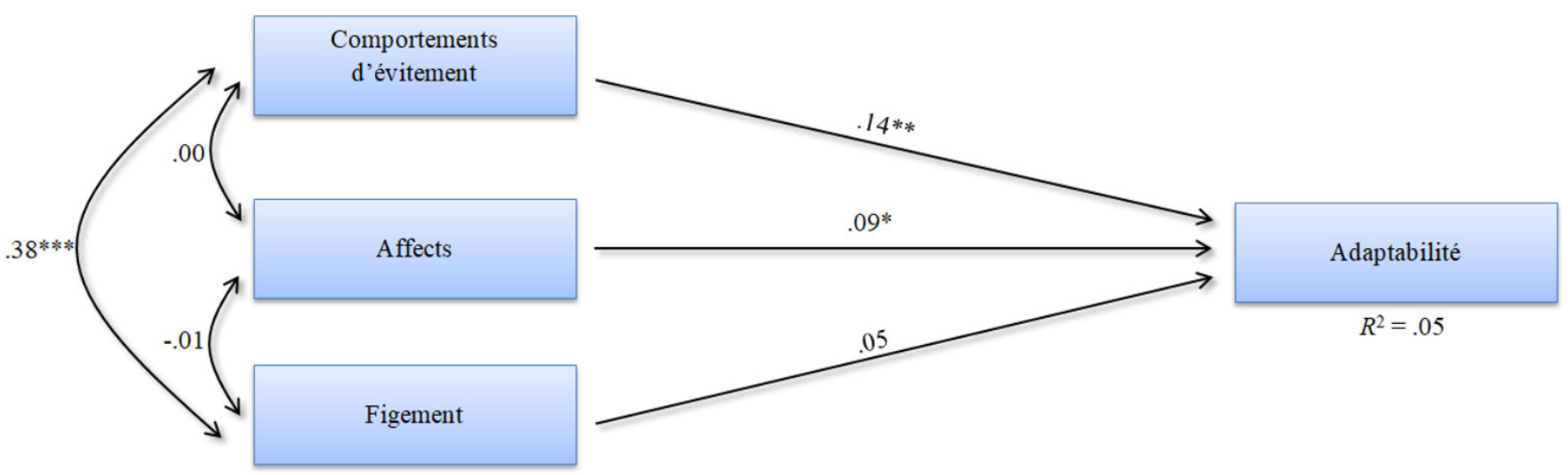

Figure 1. Modèle de régression multiple du figement, de l'évitement et des affects dans la prédiction de l'adaptabilité.

contribuent significativement à la prédiction de l'adaptabilité alors que le figement n'y contribue pas significativement tel qu'illustré dans la Figure 1. II est possible que le figement ne soit pas l'un des éléments considérés par la mère pour évaluer l'adaptabilité de son enfant puisqu'il semble passif au contraire des comportements apparents (affects négatifs ou retour à une figure familière) qu'on retrouve dans l'inhibition comportementale.

Finalement, les résultats obtenus par la méthode de jumeaux, illustrés dans la Figure 2, montrent une héritabilité du figement approximativement de $72 \%$. II semble ainsi que des facteurs génétiques prédisposent l'enfant au figement face à des situations nouvelles. Cette étude permet une meilleure compréhension de l'étiologie génétique du figement ainsi que de son lien avec l'inhibition comportementale.

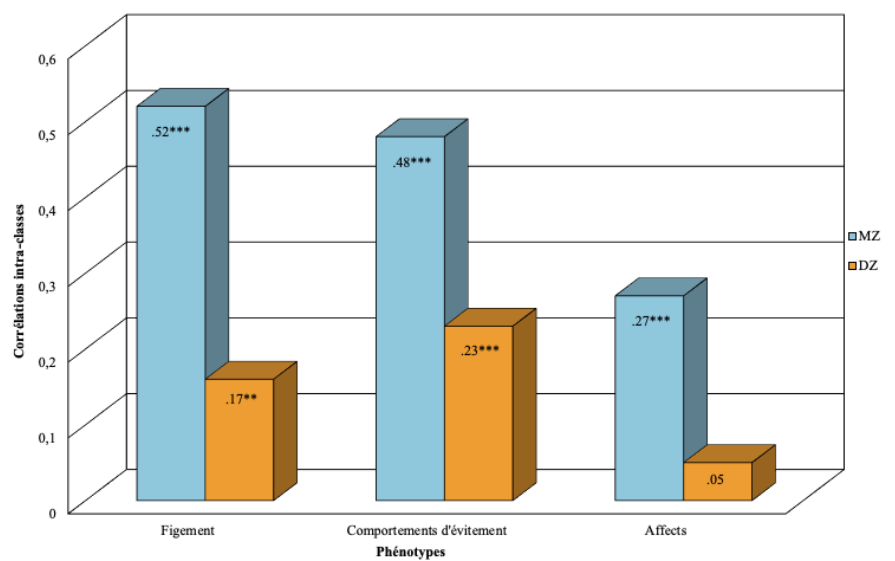

Figure 2. Corrélations intra-classes par zygotie pour le figement, les comportements d'évitement et les affects.

Note. $\mathrm{MZ}=$ monozygote $; \mathrm{DZ}=$ dizygote.

\section{Références}

Bates, J. E., Freeland, C. A., \& Lounsbury, M. L. (1979). Measurement of infant difficultness. Child Development, 50(3), 794-803. https://doi.org/10.2307/1128946

Buss, K. A. (2011). Which fearful toddlers should we worry about? Context, fear regulation, and anxiety risk. Developmental Psychology, 47(3), 804-819. https://doi. org/10.1037/a0023227

Garcia Coll, C., Kagan, J., \& Reznick, J. S. (1984). Behavioral inhibition in young children. Child Development, 55(3), 1005-1019. https://doi.org/10.2307/1130152

Hagenaars, M. A., Oitzl, M., \& Roelofs, K. (2014). Updating freeze: Aligning animal and human research. Neuroscience \& Biobehavioral Reviews, 47, 165-176. https://doi. org/10.1016/j.neubiorev.2014.07.021

Kagan, J., Reznick, J. S., Clarke, C., Snidman, N., \& Garcia-Coll, C. (1984). Behavioral inhibition to the unfamiliar. Child Development, 55(6), 2212-2225. https://doi. org/10.2307/1129793

Niermann, H. C. M., Tyborowska, A., Cillessen, A. H. N., Donkelaar, M. M., Lammertink, F., Gunnar, M. R., ... Roelofs, K. (2018). The relation between infant freezing and the development of internalizing symptoms in adolescence: A prospective longitudinal study. Developmental Science, 22(3), e12763. https://doi.org/10.1111/desc.12763

Roelofs, K. (2017). Freeze for action: neurobiological mechanisms in animal and human freezing. Philosophical Transactions of the Royal Society B-Biological Sciences, 372(1718), 10. https://doi.org/10.1098/rstb.2016.0206

\section{Pour citer l'article}

Gravel, J., Éthier, A., Provost, L., \& Boivin, M. (2020). Relation entre le figement et l'inhibition comportementale chez le jeune enfant. Psycause: Revue scientifique étudiante de l'École de psychologie de I'Université Laval, 10(2), 23-24. 